\title{
A Deeper Understanding of Quantum Thermal Machines
}

\author{
A new theoretical description of how thermal machines work in the \\ quantum regime provides a guide to increasing their efficiency. \\ By Erika K. Carlson
}

$\checkmark$ ust as classical computers need refrigeration, quantum computers will need to be cooled by thermal machines that operate in the quantum regime. Now, Bibek

Bhandari, a graduate student at the Scuola Normale Superiore di Pisa, Italy, and colleagues have developed a deeper general understanding of heat transport that could one day improve the performance of such quantum thermal machines [1].

A thermal machine, such as a refrigerator or a heat engine, transports heat between two thermal reservoirs. Two major factors determine how that heat is transported: the thermal gradient between the reservoirs and the driving forces on the machine. Previously, researchers knew that when a quantum thermal machine is driven adiabatically, the heat generated during operation can be described by the symmetric component of a geometric tensor-elements of the tensor that remain unchanged when indices that denote the element are swapped. In the new work, Bhandari and colleagues find that the conversion of heat to useful work can be described by the

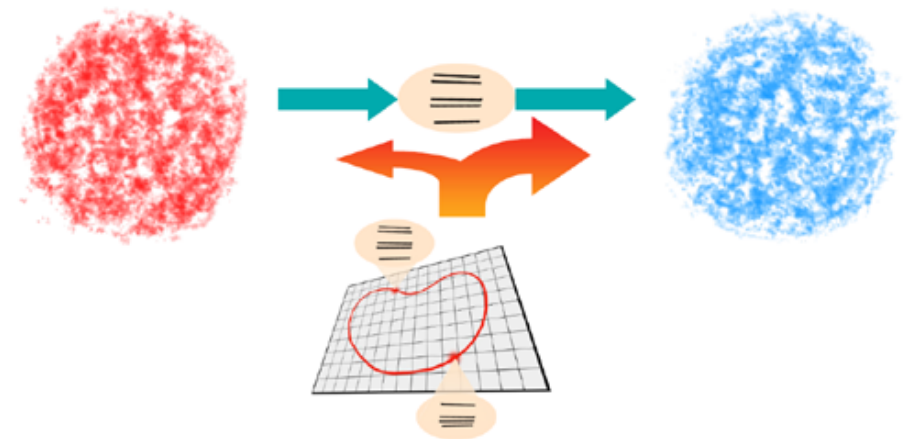

antisymmetric component of this geometric tensor-those elements that switch sign when two indices are swapped. The symmetric component is associated with dissipation of heat and is always positive because entropy always increases. But the antisymmetric component, the conversion of heat to work, can partially compensate for this energy loss. Thus designing a machine to maximize this component would minimize the entropy increase and maximize the efficiency of the machine.

The researchers used their insight to show how one might pump heat from one thermal reservoir to another in two kinds of quantum systems: a qubit and a quantum dot. Both examples, the researchers say, could be readily tested in experiments.

Erika K. Carlson is a Corresponding Editor for Physics based in New York City.

\section{REFERENCES}

1. B. Bhandari et al., "Geometric properties of adiabatic quantum thermal machines," Phys. Rev. B 102, 155407 (2020).

Credit: B. Bhandari et al. [1] 